\title{
Novel Potentiators for Vancomycin in the Treatment of Biofilm- Related MRSA Infections via a Mix and Match Approach
}

\author{
Arno Vermote, ${ }^{\dagger}$ Gilles Brackman, ${ }^{\ddagger}$ Martijn D. P. Risseeuw, ${ }^{\dagger}$ Davie Cappoen, ${ }^{\S}$ Paul Cos ${ }^{\S}$ Tom Coenye, $^{\dagger}$ \\ and Serge Van Calenbergh ${ }^{*} \dagger+(1)$ \\ ${ }^{\dagger}$ Laboratory for Medicinal Chemistry, Ghent University, Ottergemsesteenweg 460, B-9000 Ghent, Belgium \\ ${ }^{\ddagger}$ Laboratory for Pharmaceutical Microbiology, Ghent University, Ottergemsesteenweg 460, B-9000 Ghent, Belgium \\ ${ }^{\S}$ Laboratory for Microbiology, Parasitology and Hygiene, University of Antwerp, Universiteitsplein 1 (S7), B-2610 Wilrijk, Belgium
}

Supporting Information

ABSTRACT: A library of 52 hamamelitannin analogues was synthesized and investigated for its ability to potentiate the effect of vancomycin toward Staphylococcus aureus biofilms. Several compounds were found to effectively increase the susceptibility of staphylococcal biofilms toward this glycopeptide. The most active analogue identified in this study showed an $\mathrm{EC}_{50}$ value of $0.26 \mu \mathrm{M}$.

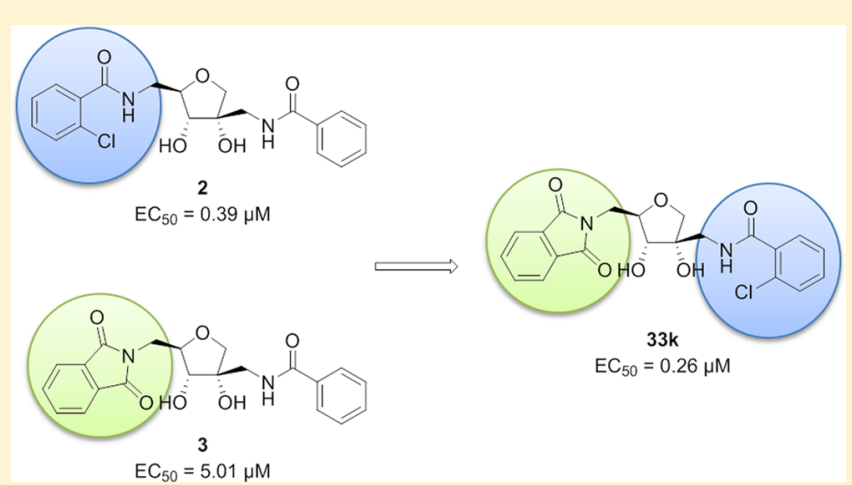

$\mathrm{EC}_{50}=5.01 \mu \mathrm{M}$

KEYWORDS: Antibiotic potentiators, Staphylococcus aureus, hamamelitannin analogues, quorum sensing, biofilms

Staphylococcus aureus (S. aureus) is an opportunistic pathogen and a leading cause of bacterial infection. ${ }^{1}$ Methicillin-resistant Staphylococcus aureus (MRSA) represents a nosocomial as well as a communal menace, and antimicrobial resistance (AMR) is eroding the clinical utility of existing antibiotics. Additionally, biofilm formation represents a significant impediment to treatment since it may worsen antimicrobial resistance to traditional antibiotics and impede host immune responses. ${ }^{2}$ Despite a recent surge, the discovery of novel antibacterial classes proves challenging. ${ }^{3}$ Hence, there is a significant and urgent need for additional therapies to combat bacterial pathogens.

Although the ability to mass-produce antimicrobial drugs constitutes a monumental scientific achievement of the twentieth century, conventional antibiotics inherently impose selective pressure on bacteria and cause an acceleration of resistance development. Bacteria have demonstrated resistance mechanisms to virtually every antibiotic introduced by the medical community. In that light, it may be argued that next to the traditional strategy of antibiotic discovery, the development of alternative approaches should also be considered to combat bacterial infections in the future.

One such strategy is the use of combinations of drugs, a paradigm that is clinically proven in many areas of medicine (e.g., in cancer chemotherapy and infectious diseases such as tuberculosis and HIV/AIDS). The pairing of an antibiotic with a nonantibiotic molecule that potentiates the activity of the former is extensively discussed in the literature. ${ }^{4-7}$ Although not possessing growth-inhibitory activity by themselves, smallmolecule "potentiators" can reduce antibiotic use and/or lessen the likelihood of resistance development. The prototype example is the $\beta$-lactamase inhibitor clavulanic acid, which "augments" the activity of the $\beta$-lactam antibiotic amoxicillin by inhibiting $\beta$-lactamases.

Kiran et al. previously identified the natural product hamamelitannin (HAM, 1, Figure 1) as a quorum sensing inhibitor (QSI) in S. aureus. ${ }^{8}$ The hydrolyzable tannin was later shown to increase $S$. aureus biofilm susceptibility toward vancomycin (VAN). ${ }^{9}$ Recently, we demonstrated that HAM affects peptidoglycan thickness and eDNA release through the quorum sensing receptor TraP. Notably, it operates via

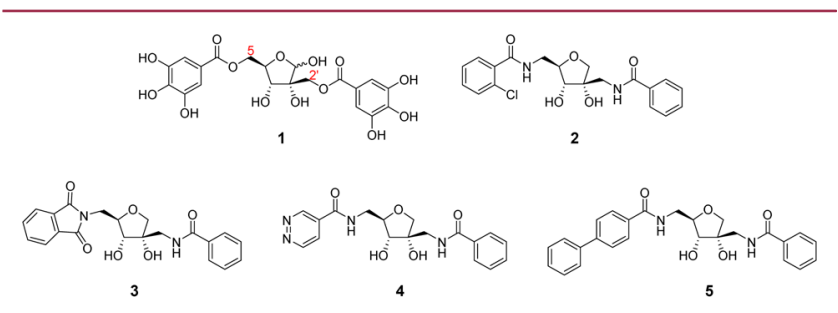

Figure 1. Structure of hamamelitannin (HAM, 1) and a selection of more drug-like derivatives $(2,3,4$, and 5$)$.

Received: August 10, 2016

Accepted: November 9, 2016

Published: November 9, 2016 
Scheme 1. Synthesis of Compounds $10 a-u$, with Differentiation at the $2^{\prime}$-Position ${ }^{a}$

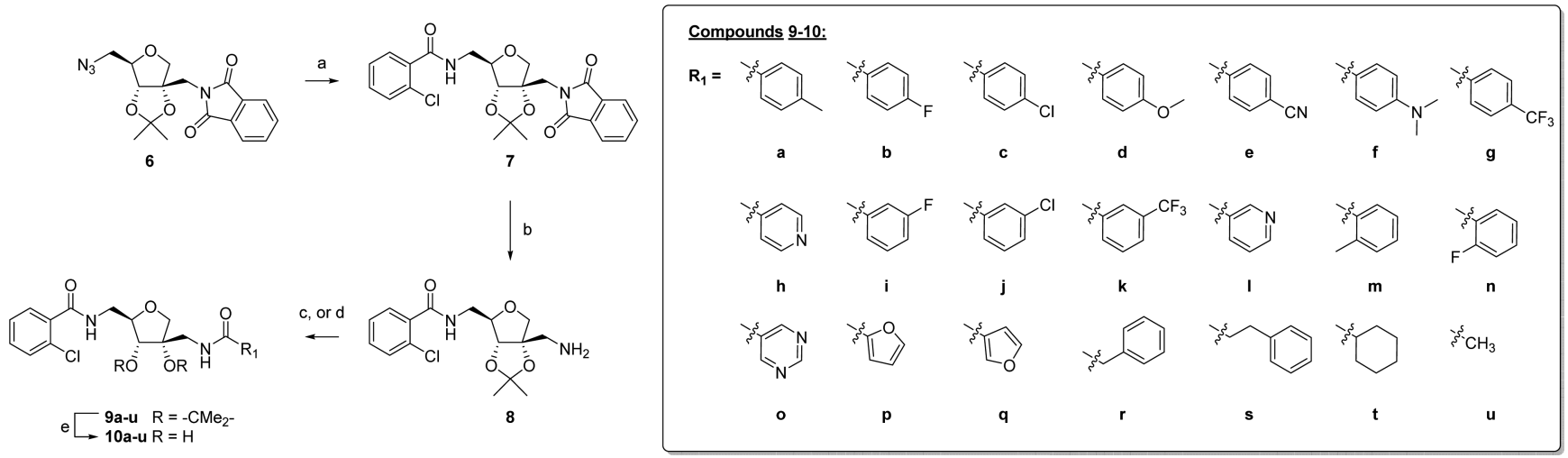

${ }^{a}$ Reagents and conditions: (a) 2-chlorobenzoyl chloride, $\mathrm{PMe}_{3}$, THF, rt, $16 \mathrm{~h}(57 \%) ;(\mathrm{b}) \mathrm{H}_{2} \mathrm{~N}_{-\mathrm{NH}_{2}} \cdot \mathrm{H}_{2} \mathrm{O}, \mathrm{EtOH}, \Delta, 5 \mathrm{~h}(82 \%)$; (c) RCOOH, EDC. $\mathrm{HCl}$, DIPEA, HOBt, DMF, rt, 16 h (68-99\%); (d) (AcO) $)_{2} \mathrm{O}$, DIPEA, $0{ }^{\circ} \mathrm{C}(71 \%)$; (e) $35 \%$ TFA in $\mathrm{H}_{2} \mathrm{O}$ (59\%-quant.).

nongrowth inhibiting mechanisms. ${ }^{10}$ Stability issues of the QSI were believed to be detrimental for further development, which led us to search for more stable analogous potentiators, ${ }^{11}$ a selection of which is shown in Figure $1(2,3,4$, and 5). The metabolically stable HAM analogue 2 (i.e., compound 38 in our previous publication ${ }^{11}$ ) was selected as a lead for further study and was shown to possess the ability to potentiate the effect of several classes of antibiotics in vitro. Moreover, the bisbenzamide increases the effect of antibiotics in two in vivo infection models.

Initial efforts to optimize the activity and stability of HAM were mainly focused on varying the 5-position of the molecule. $^{11,12}$ In the present study, we want to investigate structural variation at C-2'. Additionally, we synthesized hybrid analogues in which individual optimal aromatic moieties are combined into a single molecule in various ways.

To gain initial information on the structure-activity relationship (SAR) at the 2 '-position, we focused on benzamides with different substituents in different positions (Scheme 1). Next to that, derivatives with longer amide moieties (i.e., derived from phenyl acetic acid and phenyl propanoic acid) were synthesized. Finally, the library was extended with nonaromatic amides (i.e., an acetamide and a cyclohexanecarboxamide derivative), which allowed us to assess the role of an aromatic ring at the $2^{\prime}$-side. Note that for convenience of comparison an ortho-chlorobenzamide moiety was kept at the opposite side of the molecule. The synthesis starts from the known orthogonally protected phthalimide 6 (Scheme 1). ${ }^{11}$ One-pot azide reduction and acylation yielded intermediate 7 and avoided dimer formation due to attack of the amine on the phthalimide of a second molecule. Removal of the phthalimide with ethanolic hydrazine gave primary amine $\mathbf{8}$, which was used to generate a series of amide derivatives via EDC-mediated acylation with the appropriate carboxylic acid $(9 a-t)$. Acetamine $9 u$ was synthesized according to standard conditions. Acidic hydrolysis of the acetonide afforded the final HAM analogues 10a-u.

The minimum inhibitory concentrations (MIC) of all final compounds against $S$. aureus Mu50 were higher than $500 \mu \mathrm{M}$, indicating that they had no direct effect on growth in the concentrations used in the present study (data not shown). Subsequently, the HAM derivatives were tested for their in vitro effect on $S$. aureus biofilm susceptibility to vancomycin (VAN). VAN is considered a drug of last resort against MRSA. The biofilm susceptibility testing was done both under pretreatment and under combination treatment regimens. In the pretreatment test, $S$. aureus Mu50 was allowed to form a biofilm in the presence of a HAM analogue, after which the biofilm was treated with VAN $(20 \mu \mathrm{g} / \mathrm{mL})$. In the combination treatment setup, the bacteria were allowed to form a mature biofilm, after which VAN and a HAM derivative were administered simultaneously. S. aureus Mu50 is a clinical MRSA as well as a VISA strain (vancomycin-intermediate Staphylococcus aureus) and hence a very tenacious pathogen. ${ }^{13}$ Indeed, when used alone, VAN resulted only in a minor reduction of the number of $S$. aureus Mu50 sessile cells $(30 \pm 14 \%$ compared to an untreated control, Table 1). In contrast, combined treatment of

Table 1. Microbiological Evaluation of HAM Analogues with Differentiation at the $\mathbf{2}^{\prime}$-Position

\begin{tabular}{|c|c|c|c|c|}
\hline \multirow[b]{2}{*}{$\operatorname{compd}^{a}$} & \multicolumn{2}{|c|}{$\begin{array}{l}\text { Reduction in CFU's compared } \\
\text { to } \operatorname{ctrl}(\%)^{b}\end{array}$} & \multicolumn{2}{|c|}{$\mathrm{EC}_{50}(\mu \mathrm{M})$} \\
\hline & pretreatment & $\begin{array}{c}\text { combination } \\
\text { treatment }\end{array}$ & pretreatment & $\begin{array}{c}\text { combination } \\
\text { treatment }\end{array}$ \\
\hline $\begin{array}{l}\text { VAN } \\
\text { alone }\end{array}$ & $30 \pm 14$ & $30 \pm 14$ & & \\
\hline HAM, 1 & $35 \pm 12$ & $31 \pm 11$ & 146 & 165 \\
\hline 2 & $85 \pm 5^{c}$ & $80 \pm 4^{c}$ & 0.39 & 7.98 \\
\hline $10 a$ & $48 \pm 9$ & $58 \pm 7^{c}$ & 79.7 & 41.9 \\
\hline $10 \mathrm{c}$ & $39 \pm 3$ & $43 \pm 6$ & n.d. & n.d. \\
\hline $10 d$ & $34 \pm 18$ & $43 \pm 19$ & n.d. & n.d. \\
\hline $10 n$ & $38 \pm 18$ & $56 \pm 12^{c}$ & 48.6 & 62.2 \\
\hline 100 & $36 \pm 14$ & $35 \pm 21$ & n.d. & n.d. \\
\hline $10 q$ & $58 \pm 13^{c}$ & $50 \pm 8^{c}$ & 68.5 & 76.7 \\
\hline $10 \mathrm{~s}$ & $35 \pm 12$ & $18 \pm 22$ & n.d. & n.d. \\
\hline $10 t$ & $85 \pm 2^{c}$ & $55 \pm 7^{c}$ & 9.56 & 34.0 \\
\hline $10 \mathrm{u}$ & $70 \pm 9^{c}$ & $82 \pm 11^{c}$ & 15.4 & 3.55 \\
\hline 15 & $80 \pm 12^{c}$ & $81 \pm 8^{c}$ & 11.4 & 4.99 \\
\hline 21 & $65 \pm 10^{c}$ & $44 \pm 9$ & n.d. & n.d. \\
\hline 22 & $67 \pm 10^{c}$ & $58 \pm 15^{c}$ & n.d. & n.d. \\
\hline 23 & $94 \pm 5^{c}$ & $89 \pm 4^{c}$ & 6.32 & 8.42 \\
\hline 24 & $92 \pm 3^{c}$ & $73 \pm 8^{c}$ & 13.7 & 20.9 \\
\hline 25 & $87 \pm 4^{c}$ & $88 \pm 2^{c}$ & 15.1 & 2.34 \\
\hline
\end{tabular}

${ }^{a}$ Compounds are in addition to VAN. ${ }^{b}$ Percentage reduction in Colony Forming Units (CFU's) per biofilm when biofilms are treated with VAN alone $(20 \mu \mathrm{g} / \mathrm{mL})$ or in combination with HAM or a HAM analogue $(25 \mu \mathrm{M})$ compared to the untreated (negative) control. $c_{*}$ Significantly different from treatment with VAN alone $(p<0.05)$. n.d.: not determined. 
VAN with 2 resulted in significantly more killing of bacterial biofilm cells, both under pretreatment and under combined treatment regimens. Initially, all of the compounds with variation at the $2^{\prime}$-side of the molecule $(10 a-\mathbf{u})$ were tested in a concentration of $25 \mu \mathrm{M}$. For the most interesting derivatives, the effect on biofilm susceptibility toward VAN was also tested in lower concentrations, which allowed us to determine an $\mathrm{EC}_{50}$ value. The latter is defined as the concentration of the analogue needed to double the effect of VAN, as measured by the number of surviving cells. A full overview of the activities of the compounds from the $\mathbf{1 0}$ series is given in Table S1 (Supporting Information).

Our efforts to optimize the activity of $\mathbf{2}$ via variation of the 2 -phenyl seemed challenging. Interestingly, none of the bisbenzamides (a selection of which is given in Table 1) were exceptionally potent, with some of them being almost completely devoid of activity (e.g., 10c and 10d). In accordance with previous results, ${ }^{11}$ increasing the distance between the two aromatic rings is detrimental for activity (exemplified by $10 \mathrm{r}$ in Table S1 and 10s in Table 1). The shorter acetamide 10u, however, shows very good potentiating activity in the combination treatment setup $\left(\mathrm{EC}_{50}=3.55 \mu \mathrm{M}\right)$.

More pronounced truncation of $\mathbf{2}$ was realized through the synthesis of the "single-winged" 1-deoxyribose analogue 15 (Scheme 2). The known 5-azido-2,3-O-isopropylidene-D-

Scheme 2. Synthesis of Truncated Derivative $15^{a}$

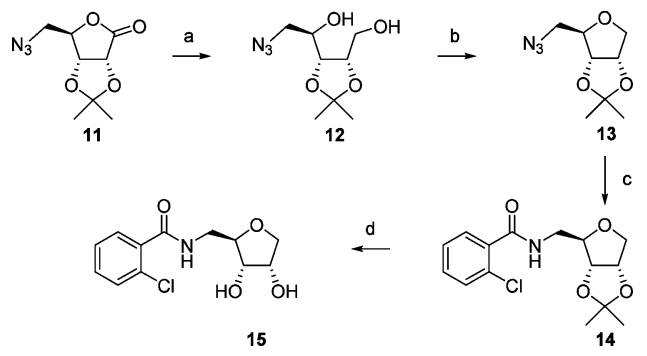

${ }^{a}$ Reagents and conditions: (a) $\mathrm{LiBH}_{4}$, THF, rt (44\%); (b) [i] $\mathrm{TsCl}$, pyr, rt, 4 h, [ii] $60{ }^{\circ} \mathrm{C}, 14 \mathrm{~h}$ (54\%); (c) [i] $\mathrm{PMe}_{3}, \mathrm{THF}, \mathrm{H}_{2} \mathrm{O}$, [ii] 2chlorobenzoic acid, EDC.HCl, DIPEA, HOBt, DMF, rt, 16 h (52\%); (d) $35 \%$ TFA in $\mathrm{H}_{2} \mathrm{O}(85 \%)$.

ribonolactone $11^{14}$ was reduced to diol 12 using $\mathrm{LiBH}_{4}$. Tosylate-promoted cyclization yielded tetrahydrofuran scaffold 13. Staudinger reduction of azide $\mathbf{1 3}$ gave the corresponding amine, which was acylated with $o$-chlorobenzoic acid and EDC as the coupling reagent. The acetal protecting group was cleaved using strong acid conditions to give final compound $\mathbf{1 5}$.

To shed light onto the role of the $2^{\prime}$-amido linker, we explored other nitrogen-based moieties to link the aromatic group to the central scaffold. Intermediate $\mathbf{8}$ served to construct analogues with alternative moieties (Scheme 3). Here again, an ortho-chlorobenzamide was installed in the 5-position. Treatment of amine $\mathbf{8}$ with benzenesulfonyl chloride gave sulfonamide 16, while treatment with phenylisocyanate yielded urea derivative 17. Imine formation of amine 8 with benzaldehyde and careful treatment with $\mathrm{NaBH}_{4}$ gave benzylamine 18. A rigidified 2'-benzamide analogue 19 was synthesized via reaction of methyl 2-bromomethyl benzoate with amine 8 . Finally, 8 was also subjected to diazotransfer to give curtailed azide 20. Removal of the acetonide in 35\% TFA in water gave final products $\mathbf{2 1 - 2 5}$ (Scheme 3).
Scheme 3. Synthesis of 21-25 with Alternative NitrogenBased Linkers $^{a}$

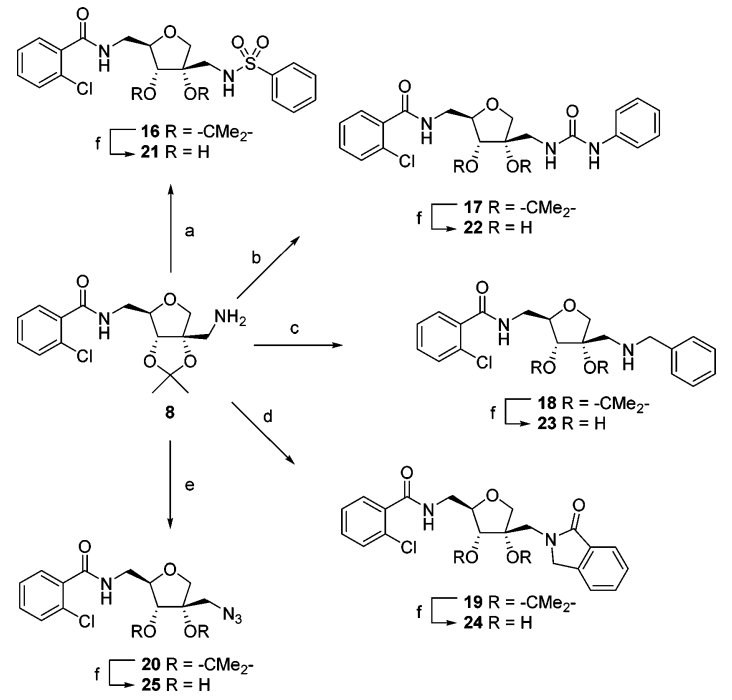

${ }^{a}$ Reagents and conditions. (a) $\mathrm{PhSO}_{2} \mathrm{Cl}, \mathrm{Et}_{3} \mathrm{~N}, \mathrm{CH}_{2} \mathrm{Cl}_{2}, 0{ }^{\circ} \mathrm{C}$ (73\%); (b) PhNCO, pyr (86\%); (c) [i] $\mathrm{PhCHO}, \mathrm{MeOH}$, mol. sieves, [ii] $\mathrm{NaBH}_{4}(65 \%)$; (d) Methyl 2-(bromomethyl)benzoate, $\mathrm{Et}_{3} \mathrm{~N}, \mathrm{MeOH}$, reflux (62\%); (e) $\mathrm{TfN}_{3}, \mathrm{MeOH}, \mathrm{Et}_{3} \mathrm{~N}, \mathrm{CuSO}_{4}(82 \%)$; (f) $35 \% \mathrm{TFA}$ in $\mathrm{H}_{2} \mathrm{O}(81-96 \%)$.

In line with what we observed for acetamide $10 \mathbf{u}$, truncated derivatives $\mathbf{1 5}$ and $\mathbf{2 5}$ also show promising potentiating activity in mature biofilms $\left(\mathrm{EC}_{50}\right.$ of 4.99 and $2.34 \mu \mathrm{M}$, respectively, Table 1). However, they lose activity in the pretreatment setup when compared to lead compound 2 . Substitution of the $2^{\prime}$ amide in $\mathbf{2}$ with a sulfonamide or a urea linker is less tolerated. Benzylamine 23, however, is performing well in the combination treatment regimen. Rigidification of $\mathbf{2}$ in the form of isoindolinone $\mathbf{2 4}$ causes a drop in activity.

It is clear from previous reports that ortho-substitution of the 5-phenyl ring (as in 2, Figure 1) increases activity, probably by distortion of the benzamide system into a nonplanar conformation. ${ }^{11,12}$ However, the more polar phthalimide 3 and pyridazine 4 (Figure 1) showed remarkably good activity. Also biphenyl derivative $\mathbf{5}$ performed well as potentiator for VAN in $S$. aureus. Nine analogues were synthesized by combining alternative 5-amide substituents (predominantly chlorinated pyridines, next to nitrogen-containing 5-rings) with a fixed $2^{\prime}$-benzamide substituent. A selection is shown in Table $2(33 \mathbf{e}-\mathbf{i})$. However, it was decided to modify both the $2^{\prime}$ - and 5 -position in a "mix and match" approach. A matrix of compounds with permutations of the different substituents was made (selection given in Table 2, i.e., 33j-y). This $\mathbf{3 3}$ series of analogues was synthesized in a manner that is essentially the same as described previously (Scheme S1 in Supporting Information). ${ }^{11} \mathrm{~A}$ full overview of all mix and match compounds is given in Figure S2 and Table S2 in the Supporting Information.

From Table 2 it is clear that there is no additive effect in combining the properties on one side of the molecule, with some of the hybrid analogues showing no potentiating activity at all (e.g., $33 \mathbf{e}-\mathbf{g})$. The permutation approach, however, afforded several active potentiators. Among these analogues, compound $33 \mathbf{k}$, which combines a 2 '-ortho-chlorobenzamide and a 5-phthalimide moiety, emerged as the strongest potentiator in both pretreatment $\left(\mathrm{EC}_{50}=0.26 \mu \mathrm{M}\right)$ and 
Table 2. Microbiological Evaluation of HAM Analogues from the Mix and Match Approach

\begin{tabular}{|c|c|c|c|c|}
\hline \multirow{2}{*}{ Compound $^{a}$} & & & \multicolumn{2}{|c|}{$\mathbf{E C}_{50}(\mu \mathrm{M})$} \\
\hline & $\mathbf{R}_{1}$ & $\mathbf{R}_{2}$ & Pretreatment & Combination treatment \\
\hline HAM, 1 & - & - & 146 & 165 \\
\hline 2 & & & 0.39 & 7.98 \\
\hline $33 e$ & & & $>250$ & 232 \\
\hline $33 f$ & & & $>250$ & $>250$ \\
\hline $33 \mathrm{~g}$ & & & 200 & $>250$ \\
\hline $33 \mathrm{~h}$ & & & 29.3 & 23.5 \\
\hline $33 \mathbf{i}$ & & & 46.7 & 58.2 \\
\hline $33 \mathbf{j}$ & & & 5.58 & 15.0 \\
\hline $33 k$ & & & 0.26 & 1.27 \\
\hline 330 & & & 5.85 & 11.4 \\
\hline $33 t$ & & & 8.54 & 45.4 \\
\hline $33 w$ & & & 24.7 & 12.1 \\
\hline $33 y$ & & & 2.63 & 39.0 \\
\hline
\end{tabular}

${ }^{a}$ Compounds are in addition to VAN.

combination treatment $\left(\mathrm{EC}_{50}=1.27 \mu \mathrm{M}\right)$ regimens. In human liver microsomal fractions, this highly potent HAM derivative is not susceptible to enzymatic degradation by UGT enzymes. It is metabolized only to a small extent through Phase I metabolism, and it was found to be stable in human plasma at $37{ }^{\circ} \mathrm{C}$ (Tables S3 and S4 in Supporting Information). Promising potentiating activities were observed for several compounds of the 33 series in S. aureus Mu50, a highly resistant and representative clinical isolate. We confirmed (as for compound 2 in our previous publication ${ }^{11}$ ) that this effect is not restricted to only one isolate (Figure S3 in the Supporting Information). Representative HAM derivatives $33 \mathbf{k}$ and 33o did not have a direct inhibiting or dispersing effect on biofilms by themselves (Figure S4), nor did combinations of 33k or 33o with VAN reduce the MIC of the antibiotic (Figure S5), indicating a specific antibiotic potentiating effect in $S$. aureus biofilms.

In conclusion, we have successfully developed several active small molecule potentiators for vancomycin in S. aureus. Most compounds with variation at the 2 -side of the molecule were devoid of activity. Exceptions are the truncated derivatives 10u, 15, and 25, which demonstrate promising activities in combination therapy. A mix and match approach of favorable substituents led to the identification of a new lead compound $33 \mathbf{k}$, which shows exceptionally good activity in both in vitro tests and warrants further study.

\section{ASSOCIATED CONTENT}

\section{S Supporting Information}

The Supporting Information is available free of charge on the ACS Publications website at DOI: 10.1021/acsmedchemlett.6b00315.

Experimental details and characterization data for the reported compounds, NMR spectra, and biological data (PDF) 


\section{AUTHOR INFORMATION}

\section{Corresponding Author}

*Tel: +32 (0)9 26481 24. Fax: +32 (0)9 26481 46. E-mail: serge.vancalenbergh@ugent.be.

\section{ORCID ${ }^{\oplus}$}

Serge Van Calenbergh: 0000-0002-4201-1264

\section{Author Contributions}

All authors have given approval to the final version of the manuscript.

\section{Funding}

The authors gratefully acknowledge funding by the Research Fund Flanders (FWO) and the Institute for the Promotion of Innovation through Science and Technology in Flanders (IWTVlaanderen, SBO program).

\section{Notes}

The authors declare no competing financial interest.

\section{ACKNOWLEDGMENTS}

The authors would like to thank Izet Karalic, Petra Rigole, Jérémy Dierickx, Jolien Claeys, and Jolien Scheerlinck for excellent technical assistance. We thank Kaushik L. Sake, Jolien Van Obbergen, and Francesca Girotti for assistance with the synthesis and Alexander Alex for design discussions.

\section{ABBREVIATIONS}

AMR, antimicrobial resistance; DIPEA, N,N-diisopropylethylamine; EDC, $N$-(3-(dimethylamino)propyl)- $N^{\prime}$-ethylcarbodiimide; HAM, hamamelitannin; HOBt, 1-hydroxybenzotriazole; MIC, minimum inhibitory concentration; MRSA, methicillinresistant Staphylococcus aureus; QSI, quorum sensing inhibitor; TFA, trifluoroacetic acid; TraP, target of RNAIII activating protein; UGT, uridine 5 '-diphospho-glucuronosyltransferase; VAN, vancomycin

\section{REFERENCES}

(1) Tong, S. Y. C.; Davis, J. S.; Eichenberger, E.; Holland, T. L.; Fowler, V. G. Staphylococcus aureus infections: epidemiology, pathophysiology, clinical manifestations, and management. Clin. Microbiol. Rev. 2015, 28, 603-661.

(2) Costerton, J. W.; Stewart, P. S.; Greenberg, E. P. Bacterial biofilms: a common cause of persistent infections. Science 1999, 284, $1318-1322$.

(3) Huttner, A.; Harbarth, S.; Carlet, J.; Cosgrove, S.; Goossens, H.; Holmes, A.; Jarlier, V.; Voss, A.; Pittet, D. Antimicrobial resistance: a global view from the 2013 World Healthcare-Associated Infections Forum. Antimicrob. Resist. Infect. Control 2013, 2, 1-13.

(4) Kiedrowski, M. R; Horswill, A. R. New approaches for treating staphylococcal biofilm infections. Ann. N. Y. Acad. Sci. 2011, 1241, 104-21.

(5) Worthington, R. J.; Melander, C. Combination approaches to combat multidrug-resistant bacteria. Trends Biotechnol. 2013, 31, 177184.

(6) Ramirez, M. S.; Tolmasky, M. E. Aminoglycoside modifying enzymes. Drug Resist. Updates 2010, 13, 151-171.

(7) Pieren, M.; Tigges, M. Adjuvant strategies for potentiation of antibiotics to overcome antimicrobial resistance. Curr. Opin. Pharmacol. 2012, 12, 551-555.

(8) Kiran, M. D.; Adikesavan, N. V.; Cirioni, O.; Giacometti, A.; Silvestri, C.; Scalise, G.; Ghiselli, R.; Saba, V.; Orlando, F.; Shoham, M.; Balaban, N. Discovery of a quorum-sensing inhibitor of drugresistant staphylococcal infections by structure-based virtual screening. Mol. Pharmacol. 2008, 73, 1578-1586.

(9) Brackman, G.; Cos, P.; Maes, L.; Nelis, H. J.; Coenye, T. Quorum sensing inhibitors increase the susceptibility of bacterial biofilms to antibiotics in vitro and in vivo. Antimicrob. Agents Chemother. 2011, 55, 2655-2661.

(10) Brackman, G.; Breyne, K.; De Rycke, R.; Vermote, A.; Van Nieuwerburgh, F.; Meyer, E.; Van Calenbergh, S.; Coenye, T. The quorum sensing inhibitor hamamelitannin increases antibiotic susceptibility of staphylococcus aureus biofilms by affecting peptidoglycan biosynthesis and eDNA release. Sci. Rep. 2016, 6, 20321.

(11) Vermote, A.; Brackman, G.; Risseeuw, M. D. P.; Vanhoutte, B.; Cos, P.; Van Hecke, K.; Breyne, K.; Meyer, E.; Coenye, T.; Van Calenbergh, S. Hamamelitannin analogues that modulate quorum sensing as potentiators of antibiotics against Staphylococcus aureus. Angew. Chem., Int. Ed. 2016, 55, 6551-6555.

(12) Vermote, A.; Brackman, G.; Risseeuw, M. D. P.; Coenye, T.; Van Calenbergh, S. Design, synthesis and biological evaluation of novel hamamelitannin analogues as potentiators for vancomycin in the treatment of biofilm related Staphylococcus aureus infections. Bioorg. Med. Chem. 2016, 24, 4563-4575.

(13) Hiramatsu, K.; Kayayama, Y.; Matsuo, M.; Aiba, Y.; Saito, M.; Hishinuma, T.; Iwamoto, A. Vancomycin-intermediate resistance in Staphylococcus aureus. J. Glob. Antimicrob. Resist. 2014, 2, 213-224.

(14) Simone, M. I.; Edwards, A. A.; Tranter, G. E.; Fleet, G. W. J. Carbon-branched $\delta$-tetrahydrofuran sugar amino acids (SAAs) as dipeptide isostere scaffolds. Tetrahedron: Asymmetry 2008, 19, 28872894. 